\title{
Use of the hyperelastic model for plastic materials by example of the three-bar truss
}

\author{
V. V. CHeKhov
}

\begin{abstract}
Particular features and traits of a model of large deformations applied for static analysis and design of metal structures with plasticity are considered. Foundations of the deformation theory of plasticity relevant for incompressible materials are formally generalized for the model of hyperelastic body described by equation of state in the Finger form. The behavior of the model is analyzed for the case of uniaxial tension. The collected results are used to explore a symmetrical threebar truss made of two materials. Relations describing behavior of the truss under conditions of the geometric and physical nonlinearities are obtained. Specifics arising in the analysis and design of the truss using standard structural alloys are analyzed.
\end{abstract}

\section{Introduction}

This work investigates application of the model of large deformations to the static analysis and design of metal structures, taking into account the behavior of the material in the plasticity zone. For the sake of combining the physical and geometric nonlinearities of hyperelastic materials in analysis and relevant software development, an attempt is made to generalize and extend the deformation theory of plasticity for the case of large strains by means of including it into the model of hyperelastic materials based on equation of state in the Finger form. In this case, the Finger strain measure is used. At present, there are various generalizations of the deformation theory of plasticity to finite deformations $[3,7,9,12,14]$, where other strain measures are used (very often this is the Hencky (logarithmic) strain tensor).

Received November 1, 2018.

2010 Mathematics Subject Classification. 74C15, 74K10, 74P05, 65H05, 93C30, 90C05.

Key words and phrases. Incompressible material, deformation theory of plasitcity, hyperelastic material, finite deformations, hybrid automation, symmetric three-bar truss, static loading, structural alloys, optimal design.

https://doi.org/10.12697/ACUTM.2020.24.11 
Using the deformation theory of plasticity, a widely known test model of a three-bar truss is considered. Its behavior can be examined analytically taking into account the possible occurrence of large deformations. The use of truss made of two materials and combination of the physical and geometric nonlinearities are two distinguished features of this analysis. Certain effects appear in this case that are unavailable for one-material structures.

All considerations here are limited to the model of incompressible materials.

\section{Incompressible material models for large deformations based on the state equation in the Finger form}

The incompressible material model for large deformations is used mainly in describing the behavior of elastomers [6]. Another type of incompressible materials are structural alloys working in the plasticity zone when their volume strain can be neglected relative to shape strain [11]. One of the theories describing such materials is the deformation theory of plasticity [11]. Unlike other theories of plasticity, the deformation theory defines a nonlinear connection between stress and strain tensors and, in fact, describes the equations of a nonlinear elastic body [11]. This aspect limits the capability of describing the phenomenon of plasticity. Nevertheless, it allows for an attempt to formally extend this theory to the model of hyperelastic body [13], which describes the behavior of elastomers under large deformations. This extension allows us to simulate the phenomenon of plasticity in software designed to work with hyperelastic materials. Under such circumstances the adequacy of the model would increase because the account for the plastic behavior of materials versus the linear-elastic model leads to a noticeable increase in the strain levels under equal external loads.

2.1. State equation of an isotropic elastic material in the Finger form. One of the common descriptions of the behavior of isotropic elastic materials under large deformations is the state equation in the Finger form [13]. It relates the Cauchy stress tensor and the Finger strain tensor:

$$
\mathbf{T}=2 J^{-1}\left(\Psi_{0} \mathbf{1}+\Psi_{1} \mathbf{b}+\Psi_{2} \mathbf{b}^{2}\right),
$$

where $\mathbf{1}$ is the identity (metric) tensor, $\mathbf{T}$ is the Cauchy stress tensor, $\mathbf{b}$ is the Finger [13] (or Cauchy-Euler [6]) strain measure which is the square of the stretch tensor: $\mathbf{b}=\boldsymbol{\Lambda}^{2} \equiv \boldsymbol{\Lambda} \cdot \boldsymbol{\Lambda}, J$ is volumetric multiplicity of the material particle, $\Psi_{0}, \Psi_{1}$, and $\Psi_{2}$ are functions of invariants of $\mathbf{b}$ which are specified by the used material model. This equation is a tensor polynomial of the second degree. In [6] such polinomial is referred to as the "classical tensor function". It allows to approximate an arbitrary dependence between coaxial symmetric tensors of the second rank (this is owing to the fact that the degree of any tensor polynomial can be lowered to the second one, taking into account the 
Hamilton-Cayley identity). For the case of an incompressible material, the state equation in the Finger form becomes [13]

$$
\mathbf{T}=-p \mathbf{1}+2\left(\Psi_{1} \mathbf{b}+\Psi_{2} \mathbf{b}^{2}\right),
$$

where $p$ is the unknown hydrostatic pressure (the Lagrange multiplier) which ensures the constancy of the volume of the material particle. Other forms can also be used for the state equation: e.g., using the -1 degree instead of 2 , or using other definitions of stress and strain tensors.

2.1.1. Uniaxial tension. In rectangular Cartesian coordinates, the constant volume condition is $\lambda_{x} \lambda_{y} \lambda_{z}=1$ where $\lambda_{x}, \lambda_{y}, \lambda_{z}$ are stretch values along the directions of the coordinate axes. Under axial symmetry

$$
\lambda_{y}=\lambda_{z}=\frac{1}{\sqrt{\lambda}_{x}}
$$

and the Finger strain tensor takes the form

$$
\mathbf{b}=\lambda_{x}^{2} \vec{i}_{1} \vec{i}_{1}+\lambda_{y}^{2} \vec{i}_{2} \vec{i}_{2}+\lambda_{z}^{2} \vec{i}_{3} \vec{i}_{3}=\lambda_{x}^{2} \vec{i}_{1} \vec{i}_{1}+\frac{1}{\lambda_{x}} \vec{i}_{2} \vec{i}_{2}+\frac{1}{\lambda_{x}} \vec{i}_{3} \vec{i}_{3}
$$

Hence the tensor equation (1) is transformed to the set

$$
\left\{\begin{array}{l}
T_{x}=-p+2\left(\Psi_{1} \lambda_{x}^{2}+\Psi_{2} \lambda_{x}^{4}\right) \\
0=-p+2\left(\Psi_{1} \frac{1}{\lambda_{x}}+\Psi_{2} \frac{1}{\lambda_{x}^{2}}\right)
\end{array}\right.
$$

which, after elimination of $p$ and designation $T_{x} \equiv \sigma$ and $\lambda_{x} \equiv \lambda$, is transformed to the form

$$
\sigma=2\left(\Psi_{1}\left(\lambda^{2}-\frac{1}{\lambda}\right)+\Psi_{2}\left(\lambda^{4}-\frac{1}{\lambda^{2}}\right)\right) .
$$

2.2. Incompressible elastomers. Various models can be used to describe the behavior of incompressible elastomers, e.g., the Mooney-Rivlin solid [13]

$$
\Psi_{1}=C_{1}+I_{\mathrm{b}} C_{2}, \quad \Psi_{2}=-C_{2} .
$$

Here $C_{1}$ and $C_{2}$ are constants of the material having the dimension of an elastic modulus. Under uniaxial tension $I_{\mathbf{b}}=b_{x x}+b_{y y}+b_{z z}=\lambda^{2}+\frac{2}{\lambda}$, and the dependence (3) takes the form

$$
\sigma=2\left(C_{1} \lambda+C_{2}\right)\left(\lambda-\frac{1}{\lambda^{2}}\right) .
$$




\subsection{Generalization of some state equations of incompressible ma- terial for large strain.}

2.3.1. Hooke's Law. Relations generalizing Hooke's law towards the case of large strain are obtained in [6]. They are known as the formulation of the standard materials of the $n$-th order. In a similar way, we will construct relations generalizing Hooke's law for incompressible material and consider only the ones that correspond to the equation (1).

Substitution of the linear strain tensor $\varepsilon$ instead of strain measure into the law of elasticity based on the tensor polynomial of -1 degree gives an approximation [6]:

$$
\mathbf{T} \approx\left(\frac{\partial \Phi}{\partial I_{\varepsilon}}+I_{\varepsilon} \frac{\partial \Phi}{\partial I I_{\varepsilon}}\right) \mathbf{1}-\frac{\partial \Phi}{\partial I I_{\varepsilon}} \varepsilon+I I I_{\varepsilon} \frac{\partial \Phi}{\partial I I I_{\varepsilon}} \varepsilon^{-1} .
$$

Here $\Phi$ is the strain energy function (strain energy density). Considering the components of $\varepsilon$ to be infinitesimal, let us keep only the linear terms with respect to them. Also, given the incompressibility of the material [6], let us replace $\mathbf{T}$ by $\mathbf{T}-p \mathbf{1}$. As a result, the law of elasticity takes the form

$$
\mathbf{T}-p \mathbf{1} \approx\left(\frac{\partial \Phi}{\partial I_{\varepsilon}}+I_{\varepsilon} \frac{\partial \Phi}{\partial I I_{\varepsilon}}\right) \mathbf{1}-\frac{\partial \Phi}{\partial I I_{\varepsilon}} \varepsilon .
$$

Thereby, to keep $\mathbf{T}$ linear against the components of $\varepsilon$, the strain energy function has to be

$$
\Phi=A I_{\varepsilon}^{2}+C I_{\varepsilon}+B I I_{\varepsilon} .
$$

Substitution of this to the linearized law of elasticity gives

$$
\mathbf{T}-p \mathbf{1} \approx\left(2 A I_{\varepsilon}+C+I_{\varepsilon} B\right) \mathbf{1}-B \varepsilon=(2 A+B) I_{\varepsilon} \mathbf{1}-B \boldsymbol{\varepsilon}+C \mathbf{1} .
$$

Let us compare this with the Hooke's law for incompressible materials [6]

$$
\mathbf{T}=p \mathbf{1}+2 \mu \varepsilon
$$

where $\mu$ is one of the Lame elastic constants (the shear modulus). From here we obtain $C=0$ and $B=-2 A=-2 \mu$, i.e.,

$$
\Phi=\mu I_{\varepsilon}^{2}-2 \mu I_{\varepsilon} .
$$

Now let us consider a tensor $\frac{\Lambda^{n}-1}{n}$, which is a generalization of $\varepsilon$ to large strains [6]. Substitution of its invariants

$$
I_{\frac{\Lambda^{n}-1}{n}}^{n}=\frac{1}{n}\left(I_{\Lambda^{n}}-3\right), \quad I I_{\frac{\Lambda^{n}-1}{n}}=\frac{1}{n^{2}}\left(-2\left(I_{\Lambda^{n}}-3\right)+\left(I I_{\Lambda^{n}}-3\right)\right)
$$

into the strain energy function (4) gives

$$
\Phi=\frac{\mu}{n^{2}}\left(I_{\Lambda^{n}}^{2}-2 I_{\Lambda^{n}}-2 I I_{\Lambda^{n}}+3\right) .
$$

Its derivatives with respect to the invariants of $\boldsymbol{\Lambda}^{n}$ are

$$
\frac{\partial \Phi}{\partial I_{\boldsymbol{\Lambda}^{n}}}=2 \frac{\mu}{n^{2}}\left(I_{\boldsymbol{\Lambda}^{n}}-1\right), \quad \frac{\partial \Phi}{\partial I I_{\boldsymbol{\Lambda}^{n}}}=-2 \frac{\mu}{n^{2}} .
$$


and their substitution into an expression of the law of elasticity for an incompressible isotropic material, expressed in terms of power-law measures of strain [6], leads to

$$
\mathbf{T}=n\left(\left(\frac{\partial \Phi}{\partial I_{\boldsymbol{\Lambda}^{n}}}+I_{\boldsymbol{\Lambda}^{n}} \frac{\partial \Phi}{\partial I I_{\boldsymbol{\Lambda}^{n}}}\right) \boldsymbol{\Lambda}^{n}-\frac{\partial \Phi}{\partial I I_{\boldsymbol{\Lambda}^{n}}} \boldsymbol{\Lambda}^{2 n}\right)+p \mathbf{1}
$$

and

$\mathbf{T}=n\left(\left(2 \frac{\mu}{n^{2}}\left(I_{\boldsymbol{\Lambda}^{n}}-1\right)-2 I_{\boldsymbol{\Lambda}^{n}} \frac{\mu}{n^{2}}\right) \boldsymbol{\Lambda}^{n}+2 \frac{\mu}{n^{2}} \boldsymbol{\Lambda}^{2 n}\right)+p \mathbf{1}=2 \frac{\mu}{n}\left(-\boldsymbol{\Lambda}^{n}+\boldsymbol{\Lambda}^{2 n}\right)+p \mathbf{1}$.

This is the law of elasticity for an incompressible standard material of $n$-th order. Setting here $n=2$ and replacing an arbitrary term $p$ by $-p$ gives

$$
\mathbf{T}=\mu\left(-\boldsymbol{\Lambda}^{2}+\boldsymbol{\Lambda}^{4}\right)-p \mathbf{1} .
$$

Comparison with the state equation in the Finger form (1) leads to

$$
\Psi_{1}=-\frac{\mu}{2}=-\frac{E}{6}, \quad \Psi_{2}=\frac{\mu}{2}=\frac{E}{6},
$$

where $E$ is the Young modulus of the material. In the case of uniaxial tension the equality (3) takes the form

$$
\sigma=\frac{E}{3}\left(\lambda^{4}-\lambda^{2}+\frac{1}{\lambda}-\frac{1}{\lambda^{2}}\right)
$$

2.3.2. Deformation theory of plasticity. Since the state equation in the Finger form contains only the strain tensor and does not contain the strain rate component, it is possible to adjust the deformation theory of plasticity to this equation. The relations of the deformation theory of plasticity are [11]:

- the volume strain is a linear-elastic one:

$$
\varepsilon_{0}=3 K \sigma_{0},
$$

where $\sigma_{0}$ and $\varepsilon_{0}$ are average normal stress and strain, respectively, and $K$ is the compression modulus;

- the stress and strain deviators are coaxial:

$$
\sigma_{i j}-\sigma_{0} \delta_{i j}=\frac{2}{3} \frac{\sigma_{\mathrm{i}}}{\varepsilon_{\mathrm{i}}}\left(\varepsilon_{i j}-\varepsilon_{0} \delta_{i j}\right),
$$

where $\sigma_{\mathrm{i}}$ and $\varepsilon_{\mathrm{i}}$ are the stress and strain intensities, respectively;

- the dependence between the stress and strain intensities is specified:

$$
\sigma_{\mathrm{i}}=f\left(\varepsilon_{\mathrm{i}}\right)
$$

(in the special case of linear elasticity, $\sigma_{\mathrm{i}}=3 \mu \varepsilon_{\mathrm{i}}$ ). 
Under advanced plastic deformations, the assumption of complete incompressibility is applicable [11], so we assume that material is incompressible: $\varepsilon_{0}=0, K=0$. Therefore

$$
\sigma_{i j}-\sigma_{0} \delta_{i j}=\frac{2}{3} \frac{\sigma_{\mathrm{i}}}{\varepsilon_{\mathrm{i}}} \varepsilon_{i j} \quad \text { or } \quad \mathbf{T}-\sigma_{0} \mathbf{1}=\frac{2}{3} \frac{\sigma_{\mathrm{i}}}{\varepsilon_{\mathrm{i}}} \varepsilon .
$$

Here we consider $\sigma_{i j}$ as the Cauchy stress tensor. Instead of the linear strain tensor $\varepsilon$, we consider its generalization to large deformations $\frac{\Lambda^{n}-1}{n}[6]$, thus

$$
\mathbf{T}-\sigma_{0} \mathbf{1}=\frac{2}{3} \frac{\sigma_{\mathrm{i}}}{\varepsilon_{\mathrm{i}}} \frac{\mathbf{\Lambda}^{n}-\mathbf{1}}{n}=\frac{2}{3 n} \frac{\sigma_{\mathrm{i}}}{\varepsilon_{\mathrm{i}}}\left(\boldsymbol{\Lambda}^{n}-\mathbf{1}\right) .
$$

Hence

$$
\mathbf{T}=\left(\sigma_{0}-\frac{2}{3 n} \frac{\sigma_{\mathrm{i}}}{\varepsilon_{\mathrm{i}}}\right) \mathbf{1}+\frac{2}{3 n} \frac{\sigma_{\mathrm{i}}}{\varepsilon_{\mathrm{i}}} \boldsymbol{\Lambda}^{n} .
$$

If we designate $\left(\sigma_{0}-\frac{2}{3 n} \frac{\sigma_{\mathrm{i}}}{\varepsilon_{\mathrm{i}}}\right) \equiv-p$ and specify $n=2$ or $n=4$, we get

$\mathbf{T}=-p \mathbf{1}+\frac{\sigma_{\mathrm{i}}}{3 \varepsilon_{\mathrm{i}}} \mathbf{\Lambda}^{2}=-p \mathbf{1}+\frac{\sigma_{\mathrm{i}}}{3 \varepsilon_{\mathrm{i}}} \mathbf{b} \quad$ or $\quad \mathbf{T}=-p \mathbf{1}+\frac{\sigma_{\mathrm{i}}}{6 \varepsilon_{\mathrm{i}}} \mathbf{\Lambda}^{4}=-p \mathbf{1}+\frac{\sigma_{\mathrm{i}}}{6 \varepsilon_{\mathrm{i}}} \mathbf{b}^{2}$,

from where

$$
\Psi_{1}=\frac{\sigma_{\mathrm{i}}}{6 \varepsilon_{\mathrm{i}}} \quad \Psi_{2}=0 \quad \text { or } \quad \Psi_{1}=0 \quad \Psi_{2}=\frac{\sigma_{\mathrm{i}}}{12 \varepsilon_{\mathrm{i}}} .
$$

In the uniaxial case, the dependence (3) takes the following form:

$$
\sigma=\frac{\sigma_{\mathrm{i}}}{3 \varepsilon_{\mathrm{i}}}\left(\lambda^{2}-\frac{1}{\lambda}\right) \quad \text { or } \quad \sigma=\frac{\sigma_{\mathrm{i}}}{6 \varepsilon_{\mathrm{i}}}\left(\lambda^{4}-\frac{1}{\lambda^{2}}\right),
$$

respectively, and increases quadratically for the physically linear-elastic case $\left(\sigma_{\mathrm{i}}=3 \mu \varepsilon_{\mathrm{i}}\right)$ or, respectively, in the 4 th degree (it is more likely to use $\lambda-1$ as $\varepsilon_{\mathrm{i}}$, because when using $\frac{\lambda^{n}-1}{n}$ with $n>1$, we get obviously understated curves $\sigma(\lambda))$.

2.4. Comparison of the dependencies under uniaxial tension. If we consider a physically linear model for steel and aluminum alloy (the diagrams are indicated on Figure 7 as " $1 \mathrm{c}$ " and " $1 \mathrm{~s}$ ") then the difference between the models (5) and (7) (with $\sigma_{\mathrm{i}}=3 \mu \varepsilon_{\mathrm{i}}$ ) is invisible within the strength of the materials. And, if the models are extended to large strains beyond the strength limits, as shown on Figure 1, then we see that the behavior of materials qualitatively corresponds to the Mooney-Rivlin model.

The use of generalizations of the deformation theory of plasticity (7), for example, for the steel with an idealized stress-strain diagram with linear hardening (denoted by "2c" on Figure 7) within the strength limit is shown on Figure 2 in comparison with the stress intensity diagram (reduced to the scale of stretches). It can be seen that, for $n=2$, the Cauchy stress curve practically coinsides with the stress intensity curve, while the account for geometrical nonlinearity, using $n=4$, can increase stress levels up to $5 \%$. 




Figure 1. Extensions of the Hooke's law to large strains.

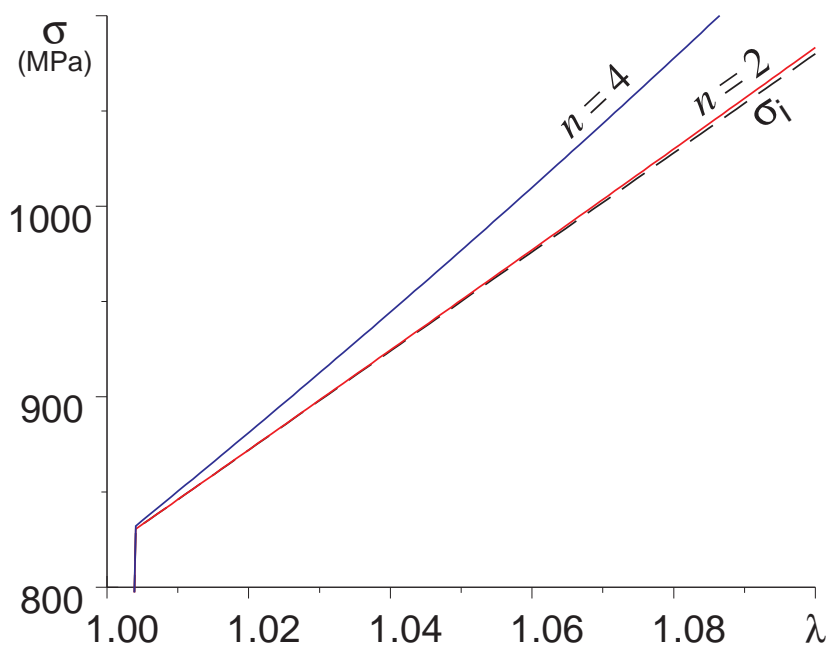

Figure 2. The uniaxial tension in steel based on the generalization of the deformation theory of plasticity.

2.5. Simulation of unloading. As it is known, the deformation theory of plasticity is inherently a theory of a nonlinear elastic body. It cannot correctly describe unloading along a path other than the loading path. However, we can add the ability to correctly describe the unloading if we use the model of hybrid automation [10]. This model combines formalisms of differential (or algebraic) equations (describing systems that are continuous in time), and a finite-state machine (describing discrete systems). For example, a model of material with linear hardening can be described as shown in Figure 3 from 



FiguRE 3. Variants of the hybrid automation for simulation hardening with unloading.

above. The work of the model within software based on hybrid automation is shown on Figure 4. If it is required to formalize a model having different diagrams for tension and compression, such a hybrid automaton will have the form shown in Figure 3 below.

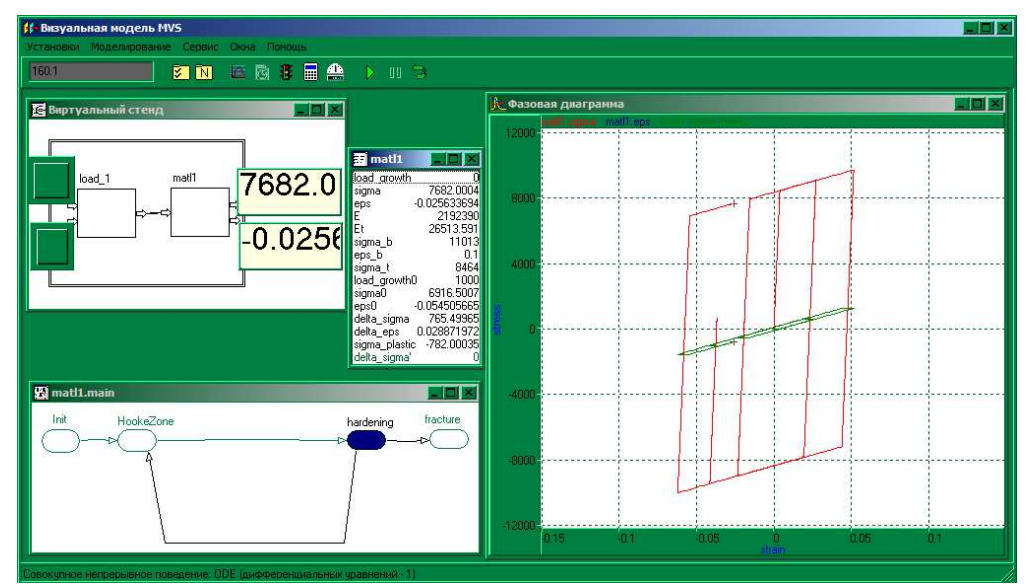

Figure 4. Simulation of the cyclic loading process according to Figure 3 from above. 


\section{A three-bar truss model}

To apply the deformation theory of plasticity for possible large deformations, a symmetric three-bar truss shown in Figure 5 is considered. The truss is symmetrically statically loaded at the point of intersection of the rods by an external constant tensile force $P$. The possible appearance of large deformation under the load is taken into account, so the initial and deformed state are assumed to be different (for comparison, the similar relations for small deformation are given). All quantities related to the initial configuration are denoted by a zero in the upper index. The angle $\alpha^{0}$ between the rods can be specified arbitrarily in the range $0 \leq \alpha^{0}<\pi / 2$. All values related to the central bar are denoted by the subscript " $c$ ", and the quantities related to the lateral bars have the subscript " $s$ ". Two incompressible materials which may be different are set in the central bar and in the lateral ones. The notation of the quantities and the scheme of the deformation of the truss are shown in Figure 6. It is obvious that only one kind of rods (central or lateral) can be deformed arbitrarily, which uniquely determines the deformation of the other $\operatorname{rod}(-\mathrm{s})$. The compatibility condition for the case of large deformations has a rather simple form.

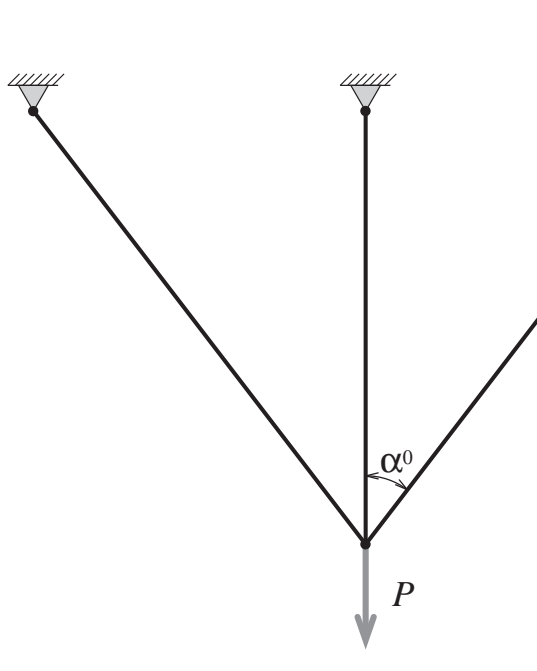

Figure 5. The 3-bar truss.



Figure 6 . The deformation of the 3-bar truss.

3.1. The compatibility condition under an arbitrary deformation. Using the notation of Figure 6, we can write the following obvious relationships:

$$
l_{c}^{0}=l_{s}^{0} \cos \alpha^{0},
$$




$$
\begin{gathered}
l_{c}=l_{s} \cos \alpha, \\
l_{c}=l_{c}^{0}+\Delta l_{c}, \\
\alpha^{0}=\alpha+\Delta \alpha, \\
l_{s}=l_{s}^{0} \cos \Delta \alpha+\Delta l_{c} \cos \alpha .
\end{gathered}
$$

By succesive elimination of the following terms from relation (12): $\Delta l_{c}$ with the aid of (10), $l_{c}$ by the use of (9), $\Delta \alpha$ by virtue of (11), and $l_{c}^{0}$ by means of (8), while carrying out the necessary transformations and reductions, we obtain

$$
\frac{l_{s}}{l_{s}^{0}}=\frac{\sin \alpha^{0}}{\sin \alpha} .
$$

Similarly, eliminating the following terms from (12) successively: $\Delta l_{c}$ with the aid of (10), $l_{s}$ by the use of (9), $\Delta \alpha$ by virtue of (11), and $l_{s}^{0}$ by means of (8), we derive an expression

$$
l_{c}=l_{c}^{0} \frac{\tan \alpha^{0}}{\tan \alpha} .
$$

These conditions can be more conveniently expressed in terms of stretch $\lambda=\frac{l}{l^{0}}$ :

$$
\lambda_{s}=\frac{\sin \alpha^{0}}{\sin \alpha}, \quad \lambda_{c}=\frac{\tan \alpha^{0}}{\tan \alpha}, \quad \frac{\lambda_{s}}{\lambda_{c}}=\frac{\cos \alpha^{0}}{\cos \alpha} .
$$

After squaring the last expression and eliminating $\sin \alpha$ from it, using the first expression, after all cancellations we derive the strain compatibility condition for the 3-bar truss:

$$
\lambda_{s}^{2}=\lambda_{c}^{2} \cos ^{2} \alpha^{0}+\sin ^{2} \alpha^{0} .
$$

If we express the compatibility condition in terms of strain $\varepsilon=\lambda-1$, then the first and second expressions (13) take the form

$$
\varepsilon_{s}=\frac{\sin \alpha^{0}-\sin \alpha}{\sin \alpha}, \quad \varepsilon_{c}=\frac{\tan \alpha^{0} \cos \alpha-\sin \alpha}{\sin \alpha} .
$$

Dividing the first expression by the second one, after some transformations, we get

$$
\frac{\varepsilon_{s}}{\varepsilon_{c}}=\frac{\sin \alpha^{0} \sin \Delta \alpha}{1+\cos \Delta \alpha}+\cos ^{2} \alpha^{0} .
$$

At small deformations $\Delta \alpha \rightarrow 0$, we have $\sin \Delta \alpha \rightarrow 0, \cos \Delta \alpha \rightarrow 1$. As a result, we obtain the well-known formulation of the compatibility condition for small deformations:

$$
\varepsilon_{s}=\varepsilon_{c} \cos ^{2} \alpha^{0}
$$


3.2. Relationship between stresses in bars and their cross-sectional areas. In projection to the axis of the central rod, the equilibrium equation for the forces at the acting load point has the following form:

$$
N_{c}+2 N_{s} \cos \alpha=P \text {. }
$$

Here $N_{c}=\sigma_{c} F_{c}, N_{s}=\sigma_{s} F_{s}$ - internal forces at the rods, $\sigma_{c}, \sigma_{s}$ - stresses at the rods, $F_{c}, F_{s}$ - the cross-sectional areas of the rods. Eliminating $\cos \alpha$ from this equation by using (13), we express the internal forces in terms of the stresses:

$$
\sigma_{c} F_{c}+2 \sigma_{s} F_{s} \frac{\lambda_{c}}{\lambda_{s}} \cos \alpha^{0}=P .
$$

Here, to use the undeformed cross-sectional areas we allow for the incompressibility condition for the uniaxial case (2), which gives $F=F^{0} / \lambda$, and then the last equation takes the form

$$
\frac{\sigma_{c}}{\lambda_{c}} F_{c}^{0}+2 \sigma_{s} \frac{\lambda_{c}}{\lambda_{s}^{2}} F_{s}^{0} \cos \alpha^{0}=P .
$$

Thus, the equation has been obtained linking the cross-sectional areas of the rods with the stresses in them.

Equation (16) connects four parameters (the cross-sectional areas and the stresses). Now, if we join it with the compatibility condition (14) (which connects the stresses - two of these parameters), then we obtain a system which allows to specify values for the two parameters and to derive values for the other two. The most simple one of the available possibilities is to set the stress in one of the bars and to set the cross-sectional area of one of the bars (the choice of the bar is arbitrary in both cases).

3.3. Evaluation of stress values for given cross-sectional areas. Using the system (14), (16), we can also solve the direct problem of calculating the stress values in rods for given areas of their cross-section. To do this, from equation (14) we express the value of one of the stretches through the other one (remark: for the current problem, the physical meaning exists when $\lambda \geq 1$ ). For example, substituting the expression of the central bar stretch in equation (16), we obtain a nonlinear equation from which we can calculate $\lambda_{s}$ :

$$
\sigma_{c}\left(\frac{\sqrt{\lambda_{s}^{2}-\sin ^{2} \alpha^{0}}}{\cos \alpha^{0}}\right) \frac{\cos \alpha^{0}}{\sqrt{\lambda_{s}^{2}-\sin ^{2} \alpha^{0}}} F_{c}^{0}+2 \frac{\sigma_{s}\left(\lambda_{s}\right) \sqrt{\lambda_{s}^{2}-\sin ^{2} \alpha^{0}}}{\lambda_{s}^{2}} F_{s}^{0}=P .
$$

Let us see now how the left side of this equation behaves. For example, let us set the cross-sectional areas of all the bars to $1 \mathrm{~cm}^{2}$ and the materials: the 30HGSA steel in the central bar and the D16 aluminium alloy in the side bars. As a model of the materials, we consider a linearly elastic $\sigma=E \varepsilon$ (with the values $E_{c}=215 \mathrm{GPa}$ and $E_{s}=72 \mathrm{GPa}$ ), and also the plastic one: with the linear hardening (passing through the points $\{0,0\},\left\{\sigma_{t} / E, \sigma_{t}\right\}$, 


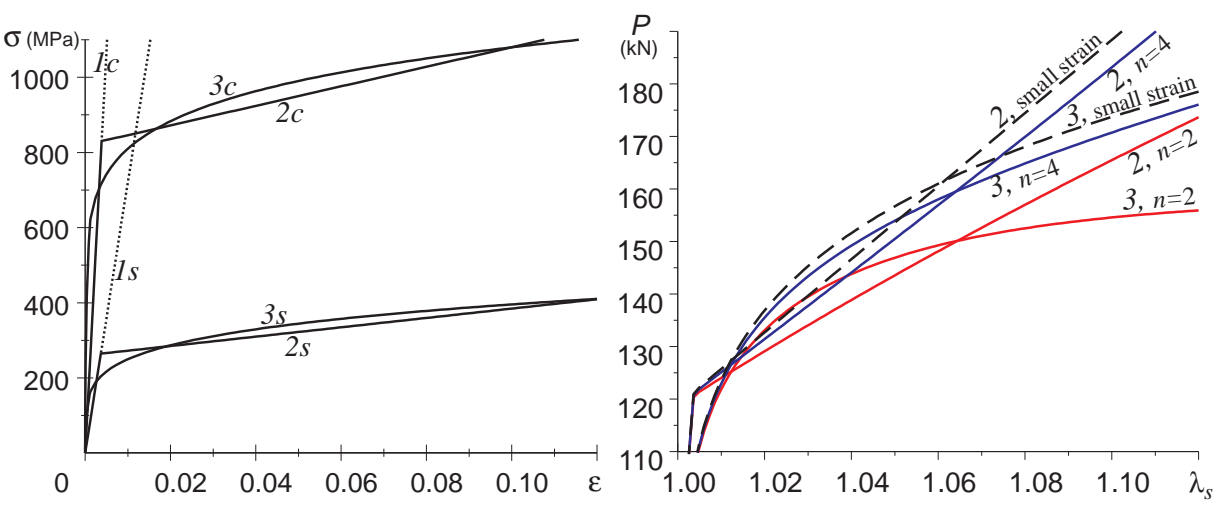

Figure 7. Stress-strain curves for steel and Al-alloy.
Figure 8. Variants of behavior of the left side of equation (17).

$\left\{\varepsilon_{b}, \sigma_{b}\right\}$ with the values $\sigma_{t c}=830 \mathrm{MPa}, \sigma_{b c}=1080 \mathrm{MPa}, \varepsilon_{b c}=0.1, \sigma_{t s}=$ $\left.265 \mathrm{MPa}, \sigma_{b s}=410 \mathrm{MPa}, \varepsilon_{b s}=0.12\right)$, and with the power approximation $\left(\sigma_{c}=1440 \varepsilon_{c}^{1 / 8}, \sigma_{s}=627 \varepsilon_{s}^{1 / 5}\right)$. The corresponding graphs are shown on Figure 7. Figure 8 demonstrates variants of behavior of the left-hand side of equation (17). Numbers 2, 3 indicate the left-hand side diagrams for the linearly hardening and power-law models of materials, respectively. The stress values were calculated using both versions of the formulas (7); the corresponding curves are denoted by $n=2$ and $n=4$.

For small deformations, equation (16) becomes

$$
\sigma_{c} F_{c}^{0}+2 \sigma_{s} F_{s}^{0} \cos \alpha^{0}=P .
$$

This equation, together with the condition of compatibility of small deformations (15), forms a set of equations, similarly to the set (14), (16) for large strain. The conclusions obtained for the set (14), (16) are also valid for this set. After eliminating the value $\varepsilon_{c}$ from (18) (by the use of (15)), we obtain a nonlinear equation with respect to $\varepsilon_{s}$. This equation is a weighted sum of stress-strain diagrams of the materials used. The behavior of the left side of this equation is shown versus the scale of stretches in Figure 8 by dashed lines. Here, the stress value was calculated as the stress intensity (6).

It can be seen that even though accounting for large deformations in the case of a single bar displays slightly higher structural stiffness (as can be seen in Figure 2), in the case of a three-bar truss, the stiffness looks underestimated under large strains, and, using the hyperelastic model, only partially approximates it to the level achieved on the basis of small deformations. For $n=2$, the curve based on the power-law model is noticeably lower than the others and, unlike them, has a region of decreasing (from $\lambda=1.2$ to 
$\lambda=4.5$ beyond the strength limit), which, apparently, is not consistent with the physical meaning of the problem).

3.4. The formulation of the optimal design problem. Consider the optimization problem for the symmetrically statically loaded three-bar truss described above (see Figure 5), taking into account the geometric and physical nonlinearities and using an incompressible material model. It is required to find the values of the cross-sectional area of the bars, which ensure the minimum of truss mass under limits on top stress levels in rods and under additonal technology constraints. The design parameters are $F_{c}^{0}$ and $F_{s}^{0}-$ the cross-sectional areas of the rods of each type. The mass value of the truss is obviously expressed in terms of the design variables:

$$
m=\rho_{c} F_{c}^{0} l_{c}^{0}+2 \rho_{s} F_{s}^{0} l_{s}^{0}=l_{c}^{0}\left(\rho_{c} F_{c}^{0}+\frac{2 \rho_{s}}{\cos \alpha^{0}} F_{s}^{0}\right),
$$

where $\rho_{c}, \rho_{s}$ are densities of the materials used (we neglect the change in density during deformation). The optimization problem is formulated as follows:

$$
\left\{\begin{array}{cl}
\rho_{c} F_{c}^{0}+\frac{2 \rho_{s}}{\cos \alpha^{0}} F_{s}^{0} \rightarrow \min _{F_{c}^{0}, F_{s}^{0}} & \\
\sigma_{c}<\bar{\sigma}_{c} ; \quad \sigma_{s}<\bar{\sigma}_{s} & \text { (stress constraints); } \\
\sigma_{c}=f_{c}\left(\lambda_{c}\right) ; \quad \sigma_{s}=f_{s}\left(\lambda_{s}\right) ; & \\
\frac{\sigma_{c}}{\lambda_{c}} F_{c}^{0}+2 \sigma_{s} \frac{\lambda_{c}}{\lambda_{s}^{2}} F_{s}^{0} \cos \alpha^{0}=P ; & \\
\lambda_{s}^{2}=\lambda_{c}^{2} \cos ^{2} \alpha^{0}+\sin ^{2} \alpha^{0} ; & \text { (tecnology constraints). }
\end{array}\right.
$$

The dependences $\sigma=f(\lambda)$ are assumed to be monotonically nondecreasing. For simplicity, the dependencies between intensities (6) combined with the stretch expression $\varepsilon=\lambda-1$ were used here.

It should be noted that even when physical and geometric nonlinearities are taken into account, equation (16) linearly connects the cross-sectional areas $F_{c}^{0}, F_{s}^{0}$ of the bars. Therefore, in a plane of the variables $F_{c}^{0}, F_{s}^{0}$, lines of equal stresses will always be straight, intersecting the coordinate axes at points

$$
\left\{F_{c}^{0}=0, \quad F_{s}^{0}=\frac{\lambda_{s}^{2}}{2 \lambda_{c}} \frac{P}{\sigma_{s} \cos \alpha^{0}}=\frac{\lambda_{s} P}{2 \sigma_{s} \cos \alpha}\right\}, \quad\left\{F_{c}^{0}=\frac{P \lambda_{c}}{\sigma_{c}}, \quad F_{s}^{0}=0\right\} .
$$

Similarly, in the case of small deformations, according to (18), the intersection points of the equal stress lines with the coordinate axes are

$$
\left\{F_{c}^{0}=0, \quad F_{s}^{0}=\frac{P}{2 \sigma_{s} \cos \alpha^{0}}\right\}, \quad\left\{F_{c}^{0}=\frac{P}{\sigma_{c}}, \quad F_{s}^{0}=0\right\} .
$$




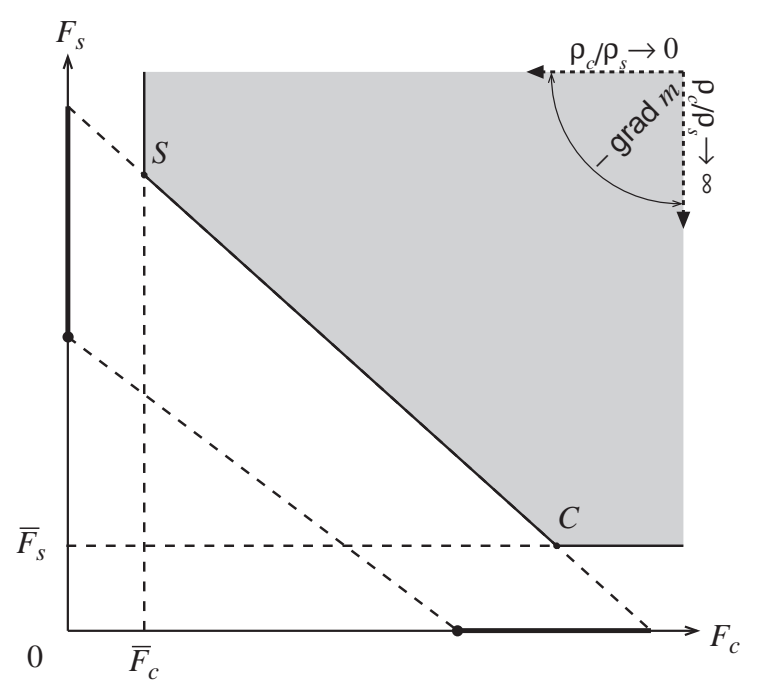

Figure 9. The feasible region and the range of the objective function gradient.

Thus, for both small and finite deformations, the optimization problem of the considered truss under nonzero technology constraints is a linear programming problem. The acceptable region and the range of possible directions for the gradient of the objective function for this problem are shown in Figure 9 (under zero technology constraints, one of the segments of the coordinate axes (shown in Figure 9 by the thick line) will be added to the acceptable region). Concrete variants of the acceptable region for real materials can be seen on a computer model of the problem [5]. Between the constraints on allowable stresses, one is active (i.e., is the strict equality), and the other is passive (being a strict inequality). Practically speaking, their simultaneous activity is very unlikely. We assume the technology constraints $\bar{F}$ to be non-zero but small enough that the active stress constraint does not overlap. Thus, in order to find the optimal design for the truss it is sufficient to consider only two solutions indicated in the figure by the letters $C$ (which have $\left.F_{c}^{0}>\bar{F}_{c}, F_{s}^{0}=\bar{F}_{s}\right)$ and $S\left(F_{c}^{0}=\bar{F}_{c}, F_{s}^{0}>\bar{F}_{s}\right)$ : one of them having a smaller mass is the optimal design. The case when the gradient of the objective function is orthogonal to the active stress constraint and the designs $C$ and $S$ have equal mass, is improbable in practice.

It should be noted that the bar length values $l_{c}^{0}, l_{s}^{0}$ are included in the relations (19) only as a part of the stretches or strains. Therefore, the problem (19) and its solution will be the same for any size of the truss (the size determines only the mass value of a specific design and not the mass ratio for different designs). 
3.5. Influence of nonlinearities on the properties of the optimal design. Let us consider how the appearance and properties of the optimal design are changed while taking into account the physical and geometric nonlinearities. Restricted to small deformations, simple examples [8] (such as pure bending or torsion of a beam, loading of the three-bar truss considered here) clearly demonstrate that, when considering the actual plastic behavior of a material, the acting internal forces are distributed more evenly compared to a linearly elastic case. As a result, the account for the physical nonlinearity shows the increased load-carrying capacity of the structure. Thereby, a design based on the linear-elastic model of the material should result in an increase of the safety margin, and, in turn, a design taking into account the plasticity of the material should reduce the mass of the optimal structure [1]. However, simple models used as a basis for these observations contain only one structural material. If more than one material is used, then an additional degree of freedom arises related to the ratio of the densities of the materials used. This degree of freedom can result in the opposite effect. As for the nature of influence of geometric nonlinearity on the properties of optimal designs, there are no simple examples that allow to draw unambiguous conclusions. Given all of the above, it is obvious that the simultaneous consideration of the physical and geometric nonlinearities should lead to the most realistic and adequate design.

Several standard structural alloys [2] were selected for the investigation. Their parameters are given in Table 1 . The properties of optimal designs were analyzed with all possible combinations of material use; i.e., altogether 49 possible options. The optimization problem (19) was considered with the following parameters: $l_{c}^{0}=1 \mathrm{~m}, \alpha^{0}=\pi / 4, P=10 \mathrm{kN}, \bar{F}_{c}=\bar{F}_{s}=$ $1 \mathrm{~mm}^{2}$, the strength limits $\bar{\sigma}$ were specified by the ultimate stresses $\sigma_{b}$. To describe the physically nonlinear behavior of materials, a model with the linear hardening was used (except for the cast iron, which, through its brittleness, was considered to be linearly elastic in all calculations).

TABLE 1. The properties of the used materials.

\begin{tabular}{l|rrrrr}
\hline Material & $\rho\left(\mathrm{kg} / \mathrm{m}^{3}\right)$ & $E(\mathrm{GPa})$ & $\sigma_{t}(\mathrm{MPa})$ & $\sigma_{b}(\mathrm{MPa})$ & $\varepsilon_{b}(\%)$ \\
\hline Bronze BrO10 & 8800 & 104 & 175 & 215 & 7 \\
Brass L75 & 8630 & 103 & 110 & 370 & 60 \\
Steel 30HGSA & 7850 & 215 & 830 & 1080 & 10 \\
Cast iron SCh35 & 7400 & 140 & - & 350 & $\sigma_{b} / E$ \\
Titanium alloy VT6 & 4450 & 115 & 1030 & 1080 & 6 \\
Aluminium alloy D16 & 2770 & 72 & 265 & 410 & 12 \\
Magnesium alloy ML5 & 1810 & 43 & 90 & 160 & 2 \\
\hline
\end{tabular}


Since this article uses an incompressible model of materials, it would be interesting to compare these results with the ones [4] obtained taking into account compressibility. Unfortunately, the source code on which [4] is based, incorrectly calculates $F_{c}^{0}$ value of the point $C$ for finite deformations (redundant divisor $\lambda_{s}$ was applied to $P$ ), so the results [4] for a geometrically nonlinear case are inaccurate. Influence of the (in)compressibility of materials on the results can be estimated visually using the simulator [5]. For the case of small deformations, the problem definition does not contain any information about the compressibility of materials, and the results of this work coincide with the ones in [4].

Combinations of materials, giving rise to the minimum or maximum mass of the optimal design, are shown in Table 2 (here and in the following tables, the used materials are enumerated with specifying the material of the central bar, and then through the dash, the material of the side bars). Table 3 shows the nature of the changes in the mass of the truss when accounting for each kind of nonlinearity. In seven cases, when the mass of the optimal design did not change when plasticity had been taken into account, the plasticity zone was not achieved due to the use of cast iron in the fully stressed element (i.e., the bar with an active stress constraint); among them, there is a rather strange design VT6-SCh35, in which a fully stressed element is simultaneously prone to degeneration. Designs where the effect of mass increase, when taking plasticity into account, is the most significant (weight increase is greater than $2 \%$ without and $10 \%$ with taking into account finite deformtions) are given in Table 4. Interestingly enough, in five of them (the first four and the last but one), taking into account the physical nonlinearity leads to the transition of the active stress constraint from the central bar to the lateral ones.

TABLE 2. Optimal designs having the smallest and largest mass value.

\begin{tabular}{l|ccc|ccr}
\hline Taking into account & \multicolumn{3}{|c|}{ Smallest mass } & \multicolumn{3}{c}{ Largest mass } \\
nonlinearities & materials & design & $m$ & materials & design & $m$ \\
\hline Lin. elast., small def. & VT6-D16 & $C$ & 47 & BrO10-BrO10 & $C$ & 428 \\
Plast., small def. & VT6-ML5 & $C$ & 46 & L75-BrO10 & $C$ & 516 \\
Lin. elast., fin. def. & VT6-D16 & $C$ & 47 & BrO10-BrO10 & $C$ & 429 \\
Plast., fin. def. & VT6-ML5 & $C$ & 48 & L75-BrO10 & $C$ & 589 \\
\hline \multicolumn{7}{c}{ including for the one-material truss } \\
\hline Lin. elast., small def. & VT6-VT6 & $C$ & 51 & BrO10-BrO10 & $C$ & 428 \\
Plast., small def. & VT6-VT6 & $C$ & 48 & BrO10-BrO10 & $C$ & 423 \\
Lin. elast., fin. def. & VT6-VT6 & $C$ & 51 & BrO10-BrO10 & $C$ & 429 \\
Plast., fin. def. & VT6-VT6 & $C$ & 50 & BrO10-BrO10 & $C$ & 451 \\
\hline
\end{tabular}


TABlE 3. Change in the mass of the optimal design when accounting for nonlinearities.

\begin{tabular}{cc|c|c|c|c|c}
\hline \multicolumn{2}{c|}{$\begin{array}{c}\text { Nonlinearity } \\
\text { under study }\end{array}$} & \multicolumn{2}{|c|}{$m_{\text {lin. }}>m_{\text {nonl. }}$} & \multicolumn{2}{|c|}{$m_{\text {lin. }}=m_{\text {nonl. }}$} & \multicolumn{2}{c}{$m_{\text {lin. }}<m_{\text {nonl. }}$} \\
other & cases & $\Delta m_{\max }$ & \multicolumn{2}{c}{ number of cases } & $\Delta m_{\max }$ \\
\hline Physical & small def. & 27 & $78 \%$ & 7 & 15 & $105 \%$ \\
& fin. def. & 19 & $78 \%$ & 7 & 23 & $133 \%$ \\
\hline Geometric & lin. elast. & 0 & - & 0 & 49 & $1 \%$ \\
& plast. & 0 & - & 0 & 49 & $54 \%$ \\
\hline \multicolumn{6}{c}{ including for the one-material truss } \\
\hline Physical & small def. & 6 & $6 \%$ & 1 & 0 & - \\
& fin. def. & 1 & $2 \%$ & 1 & 5 & $53 \%$ \\
\hline Geometric & lin. elast. & 0 & - & 0 & 7 & $1 \%$ \\
& plast. & 0 & - & 0 & 7 & $54 \%$ \\
\hline
\end{tabular}

The results given in Tables 2, 3, 4, display that taking into account the physical nonlinearity leads to an unambiguous decrease in the mass of the optimal design (within about 6\%) only for one-material structure. Meanwhile, the cases of decreasing (within 4.5 times) as well as increasing (up to 2.0 times) mass value appear already for two materials. Taking into account the geometric nonlinearity for this structure always leads to an increase in the mass of the optimal design. This is especially evident when plasticity is taken into account, hereupon strain levels increase appreciably. In many cases ( 8 out of 27) this effect overlaps the mass reduction when the plasticity is taken into account, and it occurs in 5 out of 6 cases while using one material, especially expressive is the cases L75-L75.

For the model of compressible materials, the mass growth due to the finite deformations will be slightly weaker (e.g., the maximum mass increase is $44 \%$ instead of $54 \%$ ).

TABLE 4. Optimal designs with increasing mass when accounting for physical nonlinearity.

\begin{tabular}{l|c|c|c|c|c|c}
\hline & \multicolumn{3}{|c|}{ Small deformations } & \multicolumn{3}{c}{ Finite deformations } \\
Used materials & Lin.-elast. & Plastic & $\Delta m$ & Lin.-elast. & Plastic & $\Delta m$ \\
\hline L75-BrO10 & $C(252 \mathrm{~g})$ & $C(516 \mathrm{~g})$ & $105 \%$ & $C(253 \mathrm{~g})$ & $C(589 \mathrm{~g})$ & $133 \%$ \\
$\mathrm{~L} 75-S C h 35$ & $C(246 \mathrm{~g})$ & $S(427 \mathrm{~g})$ & $74 \%$ & $C(247 \mathrm{~g})$ & $S(427 \mathrm{~g})$ & $73 \%$ \\
ML5-SCh35 & $C(130 \mathrm{~g})$ & $C(191 \mathrm{~g})$ & $47 \%$ & $C(130 \mathrm{~g})$ & $C(192 \mathrm{~g})$ & $47 \%$ \\
D16-ML5 & $C(72 \mathrm{~g})$ & $C(92 \mathrm{~g})$ & $29 \%$ & $C(72 \mathrm{~g})$ & $C(96 \mathrm{~g})$ & $34 \%$ \\
D16-SCh35 & $C(94 \mathrm{~g})$ & $C(120 \mathrm{~g})$ & $27 \%$ & $C(95 \mathrm{~g})$ & $C(120 \mathrm{~g})$ & $27 \%$ \\
30HGSA-SCh35 & $C(90 \mathrm{~g})$ & $C(111 \mathrm{~g})$ & $22 \%$ & $C(91 \mathrm{~g})$ & $C(111 \mathrm{~g})$ & $22 \%$ \\
30HGSA-ML5 & $C(77 \mathrm{~g})$ & $C(88 \mathrm{~g})$ & $15 \%$ & $C(77 \mathrm{~g})$ & $C(92 \mathrm{~g})$ & $19 \%$ \\
L75-L75 & $C(252 \mathrm{~g})$ & $C(250 \mathrm{~g})$ & $-1 \%$ & $C(252 \mathrm{~g})$ & $C(386 \mathrm{~g})$ & $53 \%$ \\
\hline
\end{tabular}


TABle 5. Difference in mass between the designs $C$ and $S$ (in $\%$ of $m_{\text {opt }}$ )

\begin{tabular}{l|cr|cr}
\hline Accounting of & \multicolumn{2}{|c}{ min. difference } & \multicolumn{2}{c}{ max. difference } \\
nonlinearities & materials & $\Delta m$ & materials & $\Delta m$ \\
\hline Lin. elast., small def. & L75-30HGSA & 65 & D16-BrO10 & 592 \\
Plast., small def. & 30HGSA-VT6 & 10 & VT6-L75 & 1842 \\
Lin. elast., finite def. & L75-30HGSA & 64 & D16-BrO10 & 590 \\
Plast., finite. def. & 30HGSA-VT6 & 3 & VT6-L75 & 1785 \\
\hline \multicolumn{2}{r}{ including for the one-material truss } \\
\hline Lin. elast., small def. & 30HGSA-30HGSA & 199 & ML5-ML5 & 282 \\
Plast., small def. & VT6-VT6 & 68 & SCh35-SCh35 & 263 \\
Lin. elast., finite def. & VT6-VT6 & 197 & ML5-ML5 & 281 \\
Plast., finite. def. & VT6-VT6 & 62 & SCh35-SCh35 & 262 \\
\hline
\end{tabular}

TABLE 6. Optimal designs with a modified geometry after taking into account the physical nonlinearity

\begin{tabular}{l|ll}
\hline \multirow{2}{*}{ Materials } & \multicolumn{2}{|c}{ Optimal design } \\
& $\begin{array}{l}\text { Linear-elastic } \\
\text { (small, finite def.) }\end{array}$ & $\begin{array}{l}\text { Plastic } \\
\text { (small, finite def.) }\end{array}$ \\
\hline BrO10-SCh35 & $C(422 \mathrm{~g}, 423 \mathrm{~g})$ & $S(424 \mathrm{~g})$ \\
BrO10-30HGSA & $C(419 \mathrm{~g})$ & $S(177 \mathrm{~g}, 178 \mathrm{~g})$ \\
BrO10-D16 & $C(413 \mathrm{~g}, 414 \mathrm{~g})$ & $S(187 \mathrm{~g})$ \\
BrO10-ML5 & $C(412 \mathrm{~g}, 413 \mathrm{~g})$ & $S(231 \mathrm{~g})$ \\
BrO10-VT6 & $C(415 \mathrm{~g}, 422 \mathrm{~g})$ & $S(91 \mathrm{~g}, 92 \mathrm{~g})$ \\
ML5-VT6 & $C(122 \mathrm{~g}, 123 \mathrm{~g})$ & $S(87 \mathrm{~g})$ \\
L75-SCh35 & $C(246 \mathrm{~g}, 247 \mathrm{~g})$ & $S(427 \mathrm{~g})$ \\
L75-30HGSA & $C(243 \mathrm{~g})$ & $S(151 \mathrm{~g}, 154 \mathrm{~g})$ \\
L75-D16 & $C(237 \mathrm{~g}, 238 \mathrm{~g})$ & $S(141 \mathrm{~g}, 144 \mathrm{~g})$ \\
L75-ML5 & $C(236 \mathrm{~g}, 237 \mathrm{~g})$ & $S(232 \mathrm{~g})$ \\
L75-VT6 & $C(239 \mathrm{~g}, 240 \mathrm{~g})$ & $S(90 \mathrm{~g})$ \\
\hline
\end{tabular}

It was analyzed how much the designs $C$ and $S$ differ in their mass for the same problem, and how realistic in practice is the optimality of the entire segment $C S$. The ranges in which there is a difference in mass between these designs are shown in Table 5 (for all designs listed here the optimum corresponds to $C$ ). It can be seen that, for two materials under both types of nonlinearities, one case turned out to be rather close to the optimality of the entire segment $C S$.

As a matter of fact, both for small and finite deformations, a linearly elastic calculation gives the optimal design $C$ in all 49 cases. However, when a physically nonlinear calculation was used, design $S$ was already optimal 
in 11 cases. This demonstrates a fundamental possibility of changing not only numerical parameters, but also the geometry of the optimal design, when physical nonlinearity is considered. The specified cases of changing the geometry are shown in Table 6 . In the linear-elastic case, the stress constraint is active only in the central bar of these designs, but, in the plastic case, it is active in the central bar in 4 cases (these are abnormal designs in which the fully stressed element is simultaneously prone to degeneration), and, in 7 cases, it is active in the side bars. Qualitatively, the same results were obtained for finite deformations (in the table, a comma-separated value of the mass relates to a corresponding geometrically nonlinear design if it differs from the geometrically linear case). Thus, the physical nonlinearity has a greater impact on the change in the configuration of the optimal design than the geometric one.

The existence of unusual designs having a fully stressed element, which is prone to degeneration, can be explained by the fact that the other (underloaded) element is made of material with large allowable stress. And, despite its underload, the last element has the stress level higher than that in the fully stressed one; i.e., this effect is due to the use of different materials in one structure.

\section{Conclusion}

The proposed generalization of the deformation theory of plasticity can expand the scope of software intended for structural analysis with hyperelastic materials. The use of this generalization in the case of uniaxial tension leads to a slight increase of stiffness versus the analysis based on the small strain model. Adequacy of this model in the plastic zone can be justified by comparison of the current results with the results obtained by other versions of the large deformations theory.

A simple model of a three-bar truss made from two materials reveals some rather interesting effects that appear in analysis and design, which takes into account geometric and physical nonlinearities.

\section{References}

[1] N. V. Banichuk, Introduction to Optimization of Structures, Springer, New York, 1990.

[2] D. V. Breslavsky, The marker of steel and alloys. http://www.splav-kharkov.com/ choose_type.php Accessed 22.07.2020. (Russian)

[3] M. Brunig, Nonlinear finite element analysis based on a large strain deformation theory of plasticity, Comput. \& Structures 69(1) (1998), 117-128.

[4] V. V. Chekhov, Geometrically and physically nonlinear optimization problem for the 3-bar truss, Dinamicheskie Sistemy (Dynamical Systems) 7(35)(2) (2017), 131-148.

[5] V. V. Chekhov, Symmetric 3-bar truss under static tension. http://vch.crimea.ua/ 3bartruss.html Accessed 22.07.2020.

[6] K. F. Chernykh and Z. N. Litvinenkova, Theory of Large Elastic Strains, LGU Publ., Leningrad, 1988. (Russian) 
[7] X.-L. Gao, Finite deformation elasto-plastic solution for the pure bending problem of a wide plate of elastic linear-hardening material, Internat. J. Solids Structures 31(10) (1994), 1357-1376.

[8] Ph. G. Hodge, Jr., Plastic Analysis of Structures, McGraw-Hill Book Co., New York, 1959.

[9] J. W. Hutchinson, Generalizing $J_{2}$ flow theory: Fundamental issues in strain gradient plasticity, Acta Mech. Sinica 28(4) (2012), 1078-1086.

[10] D. Inihov, Y. Kolesov, and Y. Senichenkov, Physical modeling of hybrid systems with rand model designer, IFAC Proceedings Volumes 45(2) (2012), 49-54.

[11] L. M. Kachanov, Fundamentals of the Theory of Plasticity, North-Holland, Amsterdam-London 1971.

[12] V. A. Lubarda, Deformation theory of plasticity revisited, The Montenegrin Academy of Sciences and Arts: Glasnik of the Section of Natural Sciences 13 (1999), 1-29.

[13] A. I. Lurie, Non-Linear Theory of Elasticity, North-Holland, Amsterdam, 1990.

[14] R. Peek, An incrementally continuous deformation theory of plasticity with unloading, Internat. J. Solids Structures 37(36) (2012), 5009-5032.

Department of Computer Science, Taurida Academy, V. I. Vernadsky Crimean Federal University, Prospekt Vernadskogo 4 , Simferopol, Republic of Crimea, 295007

E-mail address: v_chekhov@ukr.net

$U R L$ : vch.crimea.ua 